\title{
Microscopic foundation of nonextensive statistics
}

\author{
Marek Czachor ${ }^{1,2}$ and Jan Naudts ${ }^{2}$ \\ ${ }^{1}$ Katedra Fizyki Teoretycznej i Metod Matematycznych, Politechnika Gdańska ul. Narutowicza 11/12, 80-952 Gdańsk, Poland \\ ${ }^{2}$ Departement Natuurkunde, Universiteit Antwerpen, UIA, 2610 Antwerpen, Belgium
}

(Received 25 September 1998)

\begin{abstract}
A combination of the Lie-Poisson equation with the $q$-averaged energy $U_{q}=\langle H\rangle_{q}$ leads to a microscopic framework for nonextensive $q$ thermodynamics. The resulting von Neumann equation is nonlinear: $i \dot{\rho}$ $=\left[H, \rho^{q}\right]$. In spite of its nonlinearity the dynamics is consistent with linear quantum mechanics of pure states. The free energy $F_{q}=U_{q}-T S_{q}$ is a stability function for the dynamics. This implies that $q$-equilibrium states are dynamically stable. The (microscopic) evolution of $\rho$ is reversible for any $q$, but for $q \neq 1$ the corresponding macroscopic dynamics is irreversible. [S1063-651X(99)50103-7]

PACS number(s): 05.20.-y, 05.30.-d, 05.70.Ln
\end{abstract}

Standard thermodynamics is based on the GibbsShannon-von Neumann entropy $S_{1}=-k_{B} \operatorname{Tr}(\rho \ln \rho)$ and the internal energy $U_{1}=\operatorname{Tr} \rho H$. The equilibrium density matrix $\rho_{0}$ minimizes the free energy $F_{1}=U_{1}-T S_{1}$. A microscopic foundation for thermodynamics is based on the von Neumann equation (VNE)

$$
i \dot{\rho}=[H, \rho]
$$

Equilibrium states are stable fixed points of this dynamics.

It is perhaps not so widely known that the VNE can be regarded as a classical Hamiltonian system with Hamiltonian function $U_{1}$. In this context $F_{1}$ is a stability function for the underlying Hamiltonian Lie-Poisson dynamics. We shall elaborate on these points later but first we want to pose the following problem. It is known that there exist physical systems that are naturally described by a nonextensive thermodynamics [1]. The structure of this theory is analogous to the ordinary one, with one exception: Instead of $U_{1}, S_{1}$, and $F_{1}$, one takes their $q \neq 1$ generalizations $U_{q}, S_{q}$, and $F_{q}$ (see below). The question is whether there exists an underlying dynamics for nonextensive thermodynamics. Is it given by the standard VNE (as worked out in [2]) or perhaps, should the dynamics also be $q$-modified?

The analysis given below is based on one single ansatz: the $q$-averaged energy $U_{q}$ is also the Hamiltonian function of the quantum system. As we shall see, this implies that $F_{q}$ is again a stability function for the microscopic dynamics. In particular, the equilibrium states of $q$ thermodynamics are dynamically stable.

The VNE is an immediate consequence of the Schrödinger equation $(\mathrm{SE})$

$$
i|\dot{\psi}\rangle=H|\psi\rangle
$$

if $\rho$ represents a pure state, i.e., $\rho=\varrho=|\psi\rangle\langle\psi|$ (we use $\varrho$ to denote pure-state density matrices or their reductions to subsystems). However, in real experimental situations one does not deal with pure states since (a) there exists a classical lack of knowledge about quantum sources and (b) entangled states lead to nonpure density matrices when reduced to a subsystem. Indeed, if the classical state of the device that prepares the quantum ensemble is not exactly known, and the experiment is repeated several times, one has to apply a purely classical averaging over the classical configurations of the source. This results in a nonpure density matrix

$$
\rho=\int d \varrho w(\varrho) \varrho
$$

where one integrates over quantum-mechanical pure states whose distribution is given by $w(\varrho)$. $\rho$ can be used to calculate experimental averages since the latter are linear in $\varrho$ :

$$
\operatorname{Tr} \rho A=\int d \varrho w(\varrho) \operatorname{Tr} \varrho A
$$

The distinction between the state $\varrho$ and the distribution $w(\varrho)$ (a function defined on the space of states) ultimately leads to a sharp distinction between the Liouville equation (LE) and the VNE. In order to explain this point let us first note that the VNE (1) can be written as a classical Hamiltonian system [3-7]:

$$
i \dot{\rho}_{a}=\left\{\rho_{a},\langle H\rangle_{1}\right\},
$$

where $\rho_{a}=\left\langle\alpha|\rho| \alpha^{\prime}\right\rangle$ are the components of $\rho$ taken in some basis, $\langle H\rangle_{1}=\operatorname{Tr} \rho H$ is the average energy, and the bracket

$$
\{A, B\}=\rho_{a} \Omega_{b c}^{a} \frac{\delta A}{\delta \rho_{b}} \frac{\delta B}{\delta \rho_{c}}
$$

is a Lie-Poisson bracket on the manifold of states [8]. Here $\Omega_{b c}^{a}$ are structure constants of a Lie algebra $[g l(n, C)$ for finite dimensional Hilbert spaces] and the summation convention means summation or integration with respect to an appropriate measure $[9,10]$. Using the standard argument one derives the LE

$$
i \dot{w}=\left\{w,\langle H\rangle_{1}\right\}
$$

It is obvious that the linearity in $w$ of the LE (7) is completely unrelated to the linearity of the VNE, and follows directly from the fact that the dynamics (5) is Hamiltonian. Also, the physical meaning of the linearity of Eq. (7) is clear: It reflects the linearity of averaging and the fact that an experimentalist can control the form of $w$ by improving the measurement device. 
The second class of density matrices that are nonpure, in the sense that $\varrho^{2} \neq \varrho$, occurs as an entirely quantum phenomenon and is a result of entanglement between correlated quantum systems. Such states are fundamentally and irreducibly mixed. They can be written in different ways as convex combinations of pure states and all such combinations have to be regarded as physically equivalent $[11,12]$.

On the other hand, the decomposition (3) is uniquely determined by the experimental setup. The difference in the physical status of the "pure-state" decompositions of $\rho$ and $\varrho$ implies that there exists a physical difference between the linearity of the LE (7) and the VNE (1). The linearity of the latter is a postulate that is independent of both the linearity of Eq. (7) and the pure-state SE (2). Linear SE is compatible with any equation of the form

$$
i \dot{\varrho}=[H, f(\varrho)] \text {, }
$$

provided that $f(\varrho)=\varrho$ for $\varrho^{2}=\varrho$, which holds for all functions satisfying $f(0)=0$ and $f(1)=1$. The choice of $f(x)$ $=x$ is convenient but does not seem to be dictated by any fundamental principle. On the contrary, we will argue that other choices of $f$ may be physically relevant and, in particular, we will show that there exists a link of $f(x)=x^{q}$ with the nonextensive $q$ statistics introduced by Tsallis [1] [note that $q>0$ is needed to ensure $f(0)=0$ ].

We shall first show that the modified dynamics given by Eq. (8) has the same Lie-Poisson structure as (1). Assume $f$ has a Taylor expansion $f(x)=\sum_{k=1}^{\infty} f_{k} x^{k}$ with a radius of convergence of at least 1 . Consider the Lie-Poisson dynamics

$$
i \dot{\varrho}_{a}=\left\{\varrho_{a},\langle H\rangle_{f}\right\}
$$

with the one-homogeneous Hamiltonian function

$$
\langle H\rangle_{f}=\operatorname{Tr}\left\{(\operatorname{Tr} \varrho) f\left(\frac{\varrho}{\operatorname{Tr} \varrho}\right) H\right\} .
$$

A variation of $\langle H\rangle_{f}$ with respect to $\varrho$ gives the effective Hamiltonian

$$
\begin{aligned}
\hat{\mathbf{H}}(\varrho)=\frac{\delta\langle H\rangle_{f}}{\delta \varrho}= & \sum_{k=1}^{\infty} f_{k}(\operatorname{Tr} \varrho)^{1-k} \sum_{m=0}^{k-1} \varrho^{k-1-m} H \varrho^{m} \\
& +\operatorname{Tr}\left\{f\left(\frac{\varrho}{\operatorname{Tr} \varrho}\right) H\right\} \mathbf{1} \\
& -\operatorname{Tr}\left\{\frac{\varrho}{\operatorname{Tr} \varrho} f^{\prime}\left(\frac{\varrho}{\operatorname{Tr} \varrho}\right) H\right\} \mathbf{1},
\end{aligned}
$$

where $f^{\prime}=d f / d x$. Equation (9), when written in an operator notation, is

$$
i \dot{\varrho}=[\hat{\mathbf{H}}(\varrho), \varrho]=(\operatorname{Tr} \varrho)[H, f(\varrho / \operatorname{Tr} \varrho)]
$$

$\operatorname{Tr} \varrho$ is an integral of motion so we can consider solutions normalized by $\operatorname{Tr} \varrho=1$. This shows that Eq. (8) is indeed a particular case of Eq. (9). Taking $f(x)=x^{q}$ and normalized $\varrho$ we get

$$
i \dot{\varrho}=[\hat{\mathbf{H}}(\varrho), \varrho]=\left[H, \varrho^{q}\right]
$$

In general, one-homogeneity of $\langle H\rangle_{f}$ implies that

$$
\langle H\rangle_{f}=\operatorname{Tr} \varrho \hat{\mathbf{H}}(\varrho)
$$

For $f(x)=x^{q}$, denoting the corresponding $\langle H\rangle_{f}$ by $\langle H\rangle_{q}$, we find $\langle H\rangle_{q}=\operatorname{Tr} \varrho \hat{\mathbf{H}}(\varrho)=\operatorname{Tr} \varrho^{q} H$, i.e., the average effective energy equals the $q$ average of $H$, an internal energy typical of Tsallis generalized thermodynamics. Such averages were shown to be naturally linked with nonextensive $q$ entropies [13-19]. The probabilistic interpretation of $\langle H\rangle_{f}$ is derived from its equality to the "normal" average of the effective Hamiltonian. In particular, for $H=\mathbf{1}$, the generator of the dynamics is $\hat{\mathbf{H}}(\rho)=f^{\prime}(\rho)+\left[\operatorname{Tr} f(\rho)-\operatorname{Tr} \rho f^{\prime}(\rho)\right] \mathbf{1}$ which is not necessarily equal to $\mathbf{1}$. Hence there is no problem to understand why $\langle\mathbf{1}\rangle_{q} \neq 1$. The normalization of $q$ averages, as proposed recently by [20], is not desirable in the present context since the averaging we apply is essentially linear. The fact that the effective energy is "nonextensive" does not yet imply that all observables are nonextensive. On this basis one may wonder whether it is indeed physically justified to take the $q$ averaging as a universal rule for $q$ statistics.

Note that the VNE (12) is nonlinear. Nonlinear VNE's are used regularly in statistical physics. The nonlinearity is usually due to friction forces and should be compensated by adding a noise term to the VNE in order to keep the average energy constant (cf., the recent discussion in [21]). However, for a nonlinearity of the form (8) the energy is a conserved quantity. Hence there is no need for the balancing noise term. VNE's of the form (12) were independently found in the context of a Lie-Nambu dynamics and studied in [9,22]. It was shown, in particular, that their Hermitian trace-class solutions possess time-independent spectra, an important fact that allows us to treat the solutions $\varrho(t)$ as density matrices. This also implies that for any $t_{1}$ and $t_{2}$ there exists a unitary transformation satisfying $\varrho\left(t_{2}\right)=U\left(t_{2}, t_{1}\right) \varrho\left(t_{1}\right) U\left(t_{2}, t_{1}\right)^{-1}$. Although $U\left(t_{2}, t_{1}\right) \neq U\left(t_{2}-t_{1}, 0\right)$ [equality would imply linearity of evolution] the local generator of $U(t+\epsilon, t)$, for $\epsilon$ $\rightarrow 0$, exists and is our effective time-dependent Hamiltonian $\hat{\mathbf{H}}(\varrho(t))$. Denote $C_{n}=\operatorname{Tr}\left(\varrho^{n}\right), n \in N$. One finds $\left\{C_{n}, F\right\}$ $=0$ for any $F$, which shows that $C_{n}$ are Casimir invariants for the dynamics. The set of invariants also contains all $n$ averages of $H$ since $\left\{\langle H\rangle_{n},\langle H\rangle_{m}\right\}=0$ for any natural $n$ and $m$. The dynamics we consider is therefore so regular and so close to the linear one that one may wonder whether such equations do possess nontrivial solutions. Fortunately, the answer is positive. An analytic Darboux-type technique of solving Eq. (12) for $q=2$ has been recently developed $[23,24]$ and various explicit solutions were found. In a classical context the $q=2$ case was discussed in great detail in relation to Lie-algebraic generalizations of classical Euler equations (cf., Ref. [8] and references therein, in particular $[25,26])$. It is also well known that similar $q=2$ Lie-Poisson equations describe plasma dynamics (in the context of a generalized statistics, a paper of particular relevance is [27]).

Since the description we propose is meant to provide a fundamental quantum background for a generalized statistics it must be also capable of dealing with collections of nonextensive systems. This means we have to provide a recipe for 
extending the von Neumann dynamics from subsystems to composite systems. The extension should be self-consistent in the sense that a dynamics of a subsystem should be independent of whether the system is considered alone or as part of a collection of many noninteracting systems. This is achieved by taking the two-system Hamiltonian function $\left\langle H_{I}\right\rangle_{q_{1}}^{I}+\left\langle H_{I I}\right\rangle_{q_{2}}^{I I}$. The $q_{k}$ averages occurring in the Hamiltonian function of the composite system are

$$
\begin{gathered}
\left\langle H_{I}\right\rangle_{q_{1}}^{I}=\operatorname{Tr}_{I}\left[\left(\varrho_{I}\right)^{q_{1}} H_{I}\right], \\
\left\langle H_{I I}\right\rangle_{q_{2}}^{I I}=\operatorname{Tr}_{I I}\left[\left(\varrho_{I I}\right)^{q_{2}} H_{I I}\right],
\end{gathered}
$$

and the density matrices $\varrho_{I}$ and $\varrho_{I I}$ are the reduced density matrices of the respective subsystems. The two-system equation one obtains is

$$
i \dot{\varrho}_{I+I I}=\left[\hat{\mathbf{H}}_{I}\left(\varrho_{I}\right) \otimes \mathbf{1}_{I I}+\mathbf{1}_{I} \otimes \hat{\mathbf{H}}_{I I}\left(\varrho_{I I}\right), \varrho_{I+I I}\right],
$$

where $\hat{\mathbf{H}}_{I}\left(\varrho_{I}\right), \hat{\mathbf{H}}_{I I}\left(\varrho_{I I}\right)$ are the effective Hamiltonians of the subsystems. The choice of this particular type of extension follows from the general results proved for a Lie-Poisson nonlinear quantum mechanics of density matrices in $[6,9,10,28]$. The expressions $C_{n}\left(\varrho_{I+I I}\right)=\operatorname{Tr}_{I+I I}\left(\varrho_{I+I I}\right)^{n}$ are time independent (as Casimir invariants) for any natural $n$. Therefore, if $\varrho_{I+I I}(t)$ is a Hermitian trace-class solution of Eq. (14), then its eigenvalues are time independent on the basis of the standard argument [22] and, therefore, $\varrho_{I+I I}(t)$ is a density matrix if it is one at $t=0$. On the other hand, taking partial traces of Eq. (14), one verifies that

$$
i \dot{\varrho}_{I}=\left[H_{I},\left(\varrho_{I}\right)^{q_{1}}\right], \quad i \dot{\varrho}_{I I}=\left[H_{I I},\left(\varrho_{I I}\right)^{q_{2}}\right]
$$

as required by the self-consistency of the extension. All of these results have an immediate extension to more general Hamiltonian functions $\langle H\rangle_{f}$.

Having established all of these general results we are now in a position to discuss, in more detail, the links to the generalized statistics proposed by Tsallis [1]. It is based on the internal energy $U_{q}=\operatorname{Tr} \varrho^{q} H$ and the corresponding entropy

$$
S_{q}(\varrho)=k_{B} \frac{\operatorname{Tr} \varrho-\operatorname{Tr}\left(\varrho^{q}\right)}{q-1}
$$

$U_{q}$ is naturally associated with $S_{q}$ since the various relations typical of $q=1$ thermodynamics turn out to be $q$-independent. However, standard thermodynamics is static and the relations between $U_{q}$ and $S_{q}$ are evaluated in thermal equilibrium. From the dynamical point of view an equilibrium state $\varrho_{0}$ is a fixed point of the dynamics, i.e., $\left[H, \varrho_{0}\right]$ $=0$ [29]. There exists an infinite number of such states but not all of them have to be stable if a nonlinear Lie-Poisson dynamics is involved. The stability tests that are typically used in such a situation (e.g., in hydrodynamics and plasma physics) are the energy-momentum, energy-Casimir [30] or energy-invariant [31] methods. In the energy-Casimir method (used when one knows the Casimirs but does not control the symmetries) one looks for minima or maxima of the "stability function",

$$
X(\varrho)=h(\varrho)+\Phi\left(C_{1}, C_{2}, \ldots\right)
$$

where $h(\varrho)$ is a Hamiltonian function of the Hamiltonian dynamical system and $\Phi$ is a function of the Casimir invariants $C_{k}$ typical of this system. The latter function is determined by the requirement that $X(\varrho)$ has a strict minimum or maximum at $\varrho_{0}$, in particular

$$
\frac{\delta X}{\delta \varrho}\left(\varrho_{0}\right)=0
$$

In our case $h(\varrho)$ is $U_{f}=\langle H\rangle_{f}$ (or $U_{q}$ if we restrict the analysis to $\left.f(x)=x^{q}\right)$, and the Casimirs are all functions $C(\varrho)$ that can be written as a trace of a convergent power series, i.e.,

$$
C(\varrho)=\operatorname{Tr}\left(\sum_{k} c_{k} \varrho^{k}\right)=\sum_{k} c_{k} C_{k}(\varrho)=: \Phi\left(C_{1}, C_{2}, \ldots\right)
$$

It is clear that the stability function $X$ for the energy-Casimir method is nothing else but the free energy $F$ corresponding to a generalized entropy $S=-\Phi / T$, with $T$ as the temperature. In this way the thermodynamic relation $F=U_{f}-T S$ is recovered. The equilibrium state $\varrho_{0}$ is an extremum of $F$. If this is a strict minimum (or maximum) then the orbits of density matrices in a neighborhood of $\varrho_{0}$ are dynamically stable. Thermodynamic stability of the Tsallis thermodynamics has been raised in [32] and settled in [33]. Therefore equilibrium states extremizing $F$ will generically be dynamically stable fixed points of the nonlinear VNE.

Once dynamic stability of $\varrho_{0}$ is established it becomes meaningful to study linear response theory [34]. This has been done in the context of nonextensive statistics by Rajagopal $[17,35]$. However, the theory has to be modified because of the nonlinearity of the VNE. A discussion of these modifications is out of the scope of the present paper and will be presented elsewhere.

Let us illustrate the above results with the simple example of a single spin in an external field. The Hamiltonian is given by $H=-\mu \sigma_{z}$ (assume $\mu>0$, the $\sigma_{\alpha}, \alpha=x, y, z$ are the Pauli matrices). A general Hermitian $2 \times 2$ matrix with eigenvalues $\lambda_{1}, \lambda_{2}$ is

$$
\begin{aligned}
\varrho= & \frac{1}{2}\left(\lambda_{1}+\lambda_{2}\right) \mathbf{1}+\frac{1}{2}\left(\lambda_{1}-\lambda_{2}\right) \cos (\phi) \sigma_{z} \\
& -\frac{1}{2}\left(\lambda_{1}-\lambda_{2}\right) \sin (\phi)\left[\cos (\psi) \sigma_{x}+\sin (\psi) \sigma_{y}\right] .
\end{aligned}
$$

If $\sin (\phi)=0$ or $\lambda_{1}=\lambda_{2}$ then $\varrho$ is time invariant. In the other case Eq. (8) implies $\dot{\phi}=0$,

$$
\dot{\psi}=-\omega, \text { with } \omega=2 \mu \frac{f\left(\lambda_{1}\right)-f\left(\lambda_{2}\right)}{\lambda_{1}-\lambda_{2}},
$$

and the Larmor precession frequency $\omega$ depends, in general, on the eigenvalues of $\varrho$. For this example this is the only effect induced by the nonlinearity of the VNE. Note that $\omega$ $=2 \mu$ is still valid for pure states - the underlying quantum mechanics remains unchanged.

Now assume $f(x)=x^{q}$. The internal energy is

$$
U_{q}=\operatorname{Tr} \varrho^{q} H=-\mu \cos (\phi)\left(\lambda_{1}^{q}-\lambda_{2}^{q}\right) .
$$


The $q$ entropy equals $S_{q}=k_{B}\left[1-\lambda_{1}^{q}-\lambda_{2}^{q}\right] /(q-1)$ In both formulas one should take $\lambda_{1}=\lambda \geqslant 0$ and $\lambda_{2}=1-\lambda \geqslant 0$. Of physical interest are minima of $F$ for which $\lambda>1-\lambda$ and $U_{q}<0$. Hence one can take $\cos (\phi)=1$. From $\partial F / \partial \lambda=0$, with $\beta=1 / k_{B} T$ and assuming $0<|q-1| \beta \mu<1$, one finds that the eigenvalue $\lambda$ of $\varrho_{0}$ is the solution of

$$
\left(\frac{\lambda}{1-\lambda}\right)^{q-1}=\frac{1+(q-1) \beta \mu}{1-(q-1) \beta \mu} .
$$

The value of $\partial^{2} F / \partial \lambda^{2}$ at equilibrium is strictly positive. In fact, $F$ has an absolute minimum at $\varrho=\varrho_{0}$. This thermodynamic stability implies dynamic stability of $\varrho_{0}$ as a fixed point of the nonlinear VNE. One concludes that Tsallis thermodynamics is useful to analyze the dynamic stability of fixed points of the nonlinear VNE.

A special feature of the nonlinear VNE is that classical mixtures of initial conditions evolve irreversibly with time (this property has been discussed extensively in [21]). Take, e.g.,

$$
\rho=\int_{0}^{1} d \lambda \int_{0}^{\pi} \sin (\phi) d \phi \int_{0}^{2 \pi} d \psi w(\lambda, \phi, \psi) \varrho(\lambda, \phi, \psi),
$$

with $w(\lambda, \phi, \psi)=(1 / 8) \sin (\psi / 2)$ and with $\varrho(\lambda, \phi, \psi)$ given by Eq. (18). A short calculation then shows that

$$
\begin{aligned}
\rho(t)= & \frac{1}{2} \mathbf{1}+\frac{\pi}{24} \int_{0}^{1} d \lambda(2 \lambda-1) \\
& \times\left\{\cos [\omega(\lambda) t] \sigma_{x}+\sin [\omega(\lambda) t] \sigma_{y}\right\},
\end{aligned}
$$

with $\omega(\lambda)$ given by Eq. (19). Due to the dependence of $\omega$ on $\lambda$ a dephasing occurs and the classical density matrix converges to $\frac{1}{2} \mathbf{1}$ as $t \rightarrow \infty$. The lack of knowledge about initial conditions leads to a true irreversible decay.

An analysis of more complicated examples including linear response theory will be presented in a forthcoming paper.

We conclude by answering the question posed in the introduction. Nonextensive thermodynamics can be based on linear dynamics, as worked out in [2]. However, a foundation based on nonlinear dynamics is more general and more natural. In particular, it gives a probabilistic interpretation of $q$ averages. Conversely, nonextensive thermodynamics is useful to analyze the stability of nonlinear quantum systems. Finally, we have shown that nonlinearity of the dynamics can explain irreversibility of quantum systems.

We are grateful to Juan-Pablo Ortega for his comments on the stability of nonlinear Hamiltonian systems and for his interest in our work. Our collaboration is a part of the Flemish-Polish Project No. 007 and was financed in part by KBN Grant No. 2 P03B 16315.
[1] C. Tsallis, J. Stat. Phys. 52, 479 (1988).

[2] A. R. Plastino and A. Plastino, Physica A 202, 438 (1994).

[3] K. Hepp and E. H. Lieb, Helv. Phys. Acta 46, 573 (1973).

[4] P. Bóna, Comenius University Report No. Ph.10-91, 1991.

[5] N. G. Duffield and R. F. Werner, Helv. Phys. Acta 65, 1017 (1992).

[6] T. F. Jordan, Ann. Phys. (N.Y.) 225, 83 (1993).

[7] P. J. Morrison, Rev. Mod. Phys. 70, 467 (1998); I. BiałynickiBirula and P. J. Morrison, Phys. Lett. A 158, 453 (1991).

[8] J. E. Marsden and T. S. Ratiu, Introduction to Mechanics and Symmetry (Springer, New York, 1994).

[9] M. Czachor, Phys. Lett. A 225, 1 (1997).

[10] M. Czachor, Phys. Rev. A 57, 4122 (1998).

[11] B. Mielnik, in Problems in Quantum Physics, edited by J. Mizerski et al. (World Scientific, Singapore, 1990).

[12] N. D. Mermin, e-print quant-ph/9801057; this is the most detailed and clear presentation of the problem we have found in the literature.

[13] E. M. Curado and C. Tsallis, J. Phys. A 24, L69 (1991); 24, 3187 (1991); 25, 1019 (1992).

[14] F. Büyükkiliç, D. Demirhan, and A. Güleç, Phys. Lett. A 197, 209 (1995); F. Pennini, A. Plastino, and A. R. Plastino, ibid. 208, 309 (1995).

[15] C. Tsallis, Phys. Lett. A 195, 329 (1994).

[16] A. R. Plastino and A. Plastino, Phys. Lett. A 177, 177 (1993).

[17] A. K. Rajagopal, Phys. Lett. A 214, 127 (1996).
[18] C. Tsallis and A. M. C. de Souza, Phys. Lett. A 235, 444 (1997).

[19] C. Tsallis, F. C. Sá Baretto, and E. D. Loh, Phys. Rev. E 52, 1447 (1995); A. B. Pinheiro and I. Roditi, Phys. Lett. A 242, 296 (1998).

[20] C. Tsallis, R. S. Mendes, and A. R. Plastino, Physica D (to be published).

[21] M. Grigorescu, Physica A 256, 149 (1998).

[22] M. Czachor and M. Marciniak, Phys. Lett. A 239, 353 (1998).

[23] S. B. Leble and M. Czachor, Phys. Rev. E 58, 7091 (1998).

[24] M. Kuna, M. Czachor, and S. B. Leble, e-print quant-ph/9810023.

[25] M. Adler and P. van Moerbeke, Adv. Math. 38, 267 (1980).

[26] T. Ratiu, Am. J. Math. 104, 409 (1982).

[27] B. M. Boghosian, Phys. Rev. E 53, 4754 (1996).

[28] M. Czachor and M. Kuna, Phys. Rev. A 58, 128 (1998).

[29] There exists another class of stationary solutions obtained by requiring that $\hat{\mathbf{H}}(\rho)=0$. For example, if $q=2$, this amounts to $H \rho+\rho H=0$ and $\operatorname{Tr} \rho H=0$, which cannot be satisfied if both $\rho$ and $H$ are positive.

[30] D. D. Holm, J. E. Marsden, T. Ratiu, and A. Weinstein, Phys. Rep. 123, 1 (1985).

[31] J.-P. Ortega, Ph.D. thesis, University of California, Santa Cruz, 1998 (unpublished).

[32] J. D. Ramshaw, Phys. Lett. A 198, 119 (1995).

[33] C. Tsallis, Phys. Lett. A 206, 389 (1995).

[34] R. Kubo, J. Phys. Soc. Jpn. 12, 570 (1957).

[35] A. K. Rajagopal, Physica A 253, 271 (1998). 